\title{
A Rare Case of Ganglioneuroblastoma with Presentation of Diarrhea and Resistant Hypokalemia
}

\author{
Maryam Boroomand ${ }^{1}$, Hosein Alimadadi ${ }^{2}$, Mastaneh Moghtaderi*3 \\ 1 Pediatrician, Children Medical Center Hospital, Tehran University of Medical Science (TUMS), Tehran, Iran; Email: \\ m.boroomand1363@gmail.com \\ 2 Associated Professor of Pediatric Gasteroentrology, Children Medical Center Hospital, Tehran University of Medical Science (TUMS), \\ Tehran, Iran; Email: hoseinalimadadi@yahoo.com \\ 3 Associated Professor of Pediatric Nephrology, Chronic Kidney Research Center, Children Medical Center Hospital, Tehran University of \\ Medical Science (TUMS), Tehran, Iran \\ *Corresponding Author: Dr. Mastaneh Moghtaderi, Associated Professor of Pediatric Nephrology, Chronic Kidney Research \\ Center, Children Medical Center Hospital, Tehran University of Medical Science, Tehran, Iran, Tel: +989127183199, Email: \\ drmoghtaderi@gmail.com
}

Received: February 15, 2019; Published: March 28, 2019

\section{Abstract}

Background: Neuroblastoma is the most common extracranial solid tumor in children and the most common malignancy in infant. Some tumors release vasoactive intestinal peptide (VIP), causing a profound secretory diarrhea. Case Report: In this report we describe a 33 month old girl with intermittent diarrhea from 9 month ago and hypokalemia resistant to treatment .She had increased VMA in 24hour urine .After complete workup she had a mass in her thorax that resected and her diarrhea and hypokalemia was improved. Conclusion: In children with resistant diarrhea we should consider tumors secreting VIP.

Keywords: Neuroblastoma, Diarrhea, Hypokalemia.

\section{INTRODUCTION}

Neuroblastoma (NB) is the most common extracranial solid tumor in children. The site of primary tumor can be in any part of the sympathetic chain or the adrenal medulla [1]. The tumor can be found in the abdomen (75\%), mediastinum $(20 \%)$ and the neck (5\%) [2]. It can be primary or metastatic [3]. The signs are palpable mass in the abdomen or neck, loss of appetite and vomiting (in abdominal tumors), shortness of breath, pulmonary infections, and dysphagia (in Chest tumors), anemia and petechiae (in bone-marrow involvement). The initial presentation can be metastatic lesions of liver, lung or bone. Excretion of catecholamine and VIP can lead to sweating, pallor, hypertension and watery diarrhea [4]. Patients diagnosed under 1 years of age (40\%) have a much better prognosis than those diagnosed in later childhood [5]. The diagnosis is difficult because of wide range of clinical presentations. The methods to diagnosis are ultrasound, CT scan and urine VMA analysis. Biopsy from the metastatic lesions can confirm the diagnosis [6]. Depending on the stage of tumor the treatment includes surgical excision, multi agent chemotherapy, and bone marrow transplantation [6]. Patients with diarrhea as the main symptom, namely diarrheal NB that is rare [7]. Diarrheal NB is not easy to diagnose in the early stage of the disease [8]. The initial clinical manifestations are intractable diarrhea, hypokalemia, and dysplasia. In these patient's VIP has increased. Potassium supplementation plays a vital role in the treatment. On the condition of appropriate treatment, diarrheal NB has a good prognosis [8]. With surgical excision of the tumor, diarrhea resolute completely [9].

\section{CASE REPORT}

We are reporting a 33-month-old girl with the complaint of intermittent diarrhea from 9 month ago. Her diarrhea was watery and voluminous, 3-4 times a day. Also, she had sometimes vomiting and abdominal pain and blowing. She had 4 kilogram decrease in weight duration of 9 month. In laboratory tests she had hypokalemia and hyponatremia resistant to treatment. Her diarrhea was improved with NPO and Octerotide. Her sonography revealed distended loops with much fluid. In her 24hour urine had increased VMA. In her endoscopy esophagus and stomach and D1 were normal but D2 had decrease in the size of villus and scalloping in some points. In her colonoscopy had nodularity in descending colon and decrease of vascular pattern. A chest CT was performed for her. The findings were a large posterior mediastinal right paravertebral soft tissue mass 
about $49 \times 41 \times 65 \mathrm{~mm}$ with fine calcified foci, minimal reactive pleural effusion without evidence of lung involvement that was most likely suspected mediastinal neuroblastoma. In MRI the mass was hyposignal in T1 and hypersignal in T2 with spreading to spinal cord from neural foraments that had changed the site of techal sac but had not pressure effect on the neural cord. At the end the mass was excited totally from right thoracotomy and her diarrhea and hypokalemia was improved. The pathology of the tumor was ganglioneuroblastoma.
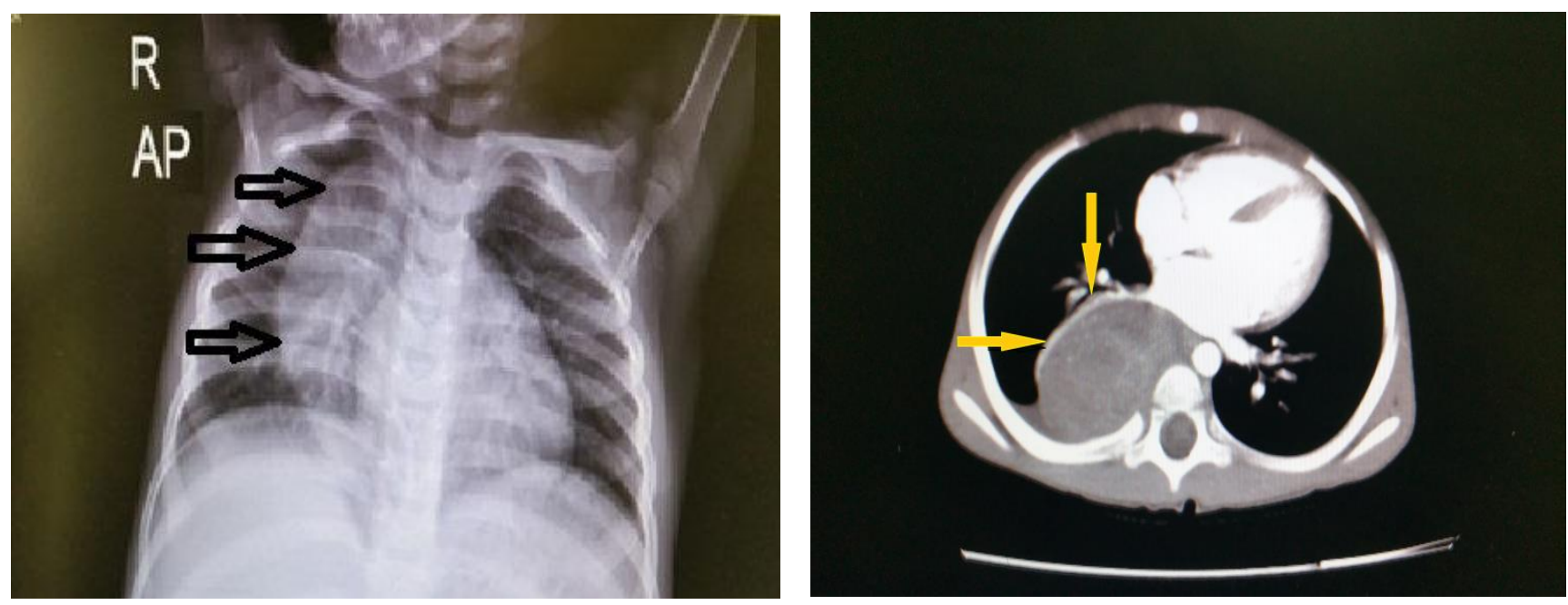

Figure 1: large posterior mediastinal right paravertebral soft tissue mass with fine calcified foci, minimal reactive pleural effusion and no evidence of lung involvement

\section{DISCUSSION}

Neuroblastoma (NB) is the most common extracranial solid tumor in children. It can be found in the abdomen (75\%), mediastinum (20\%) and the neck (5\%) [2]. It can be primary or metastatic [3]. Fever, limb pain, abdominal mass, abdominal pain, and lower limb weakness are the most common clinical manifestations in the most patients. Diarrheal NB is rare and it is not easy to diagnose in the early stage of the disease[8]. Secretion of large amounts of VIP leads to Intractable diarrhea, hypokalemia, and achlorhydria [11]. such as our patient. In 1952, Hawfield and Daisly reported a case of adrenal neuroblastoma with chronic diarrhea that it improved after removal of the tumor [12]. Other investigators also reported ganglioneuroma and ganglion NB with watery diarrhea, hypokalemia, and achlorhydria syndrome in 1973 [13]. VIP secreting tumors include pheochromocytoma, mast cell tumors, non B cell hyperplasia, and medullary thyroid carcinoma. Diarrheal NB was first reported by Ghishan et al [14] in 1979. VIP secreting tumors characterized by watery diarrhea, hypokalemia, and alkalosis (WDHA syndrome). Our patient had not alkalosis. This syndrome is most common in children aged one year to three years, and should be included in the differential diagnosis of pediatric chronic diarrhea. VIP was first isolated in 1970 from pig small intestines [15.] The central nervous system and the sympathetic ganglion neurons are also rich in VIP[8]. VIP is a polypeptide of 28 amino acid residues. It stimulates intestinal water and electrolyte secretion by activating adenylate cyclase and cyclic AMP in intestinal cells [16]. Also, it is distributed in the digestive tract, the central and peripheral nervous systems.[17] VIP also inhibits gastric acid secretion, promotes hepatic glycogenolysis and hyperglycemia and dilates peripheral systemic blood vessels [18]. The signs can be secretory diarrhea, dehydration, and flushing and weight loss. [19] Our case also had these signs. Laboratory abnormalities include hypokalemia, achlorhydria and metabolic acidosis $[19,20]$. In resect able stage1 (confined to the site of origin) and stage 2 (extending beyond the site of origin but not crossing the midline), surgery alone is usually sufficient without the need for chemotherapy or radiotherapy. In stage 3 (tumor extending beyond the midline) and stage 4 (remote metastases to bones or lymph nodes), chemotherapy and/or radiotherapy are required. Our case was in stage one of tumor and improved after excision of the tumor. We suggest that in a patient with chronic diarrhea without any reason, VIP-secreting tumor should be considered and gastrointestinal, abdominal and thoracical ultrasound or CT scan were done to discover a mass. The serum level of VIP should be measured. Ganglioneuroblastoma and ganglioneuroma are the commonest tumors in children.

\section{CONCLUSION}

Ganglioneuroblastoma may have many atypical presentations, so awareness and keeping it in mind have dratistic importance in early detection and preventing of its serious consequences.

\section{Conflict of Interest}

None declared

\section{Financial Support}

None declared

\section{REFERENCES}

1. Ganeshan VR, Schor NF. Pharmacologic management of high risk neuroblastoma in children. Pediatric Drugs 2011; 13: 245255.

2. Maris JM. Recent advances in neuroblastoma. $\mathrm{N}$ Engle $\mathrm{J}$ Med 2010; 362: 2202-2211.

3. Simpson JK, Gaze MN. Current Management of Neuroblastoma. 
Oncologist 1998; 3: 253-62.

4. Dupont C. Vipomas. Ann Med Interne (Paris) 1984; 135: 400-02.

5. Lee KL, Ma JF, Shortlife LD. Neuroblastoma management, recurrence, and follow-up. Urol Clin N Am., 2003; 30: 881-89

6. Shreya Shah, Nisarg Champaneria, RK Pasle. Neuroblastoma in 3 months old infant - A rare case report ; IAIM, 2016; 3(7): 362365.

7. Mendelsohn G, Eggleston JC, Olson JL, Said SI, Baylin SB. Vasoactive intestinal peptide and its relationship to ganglion cell differentiation in neuroblastic tumors. Lab Invest 1979; 41: 144-149.

8. Wei Han, Huan-Min Wang. Refractory diarrhea: A paraneoplastic syndrome of neuroblastoma ; World J Gastroenterol 2015 July 7; 21(25): 7929-7932.

9. Craig $M$ Lofthouse, Anthony $K$ Akobeng, Jenny Adamski, Bernadette Brennan .A 2-year-old boy with diarrhoea and failure to thrive; Lancet 2003; 361: 1012.

10. Gilshtein H, Peled Z, Grunner S, Fischer D, Kakiashvili E, Kluger Y. Ganglioneuroma of the adrenal gland: a rare tumor in a rare location. Case Rep Oncol 2012; 5: 487-489.

11. Wildhaber B, Niggli F, Bergsträsser E, Stallmach T, Sacher P. Paraneoplastic syndromes in ganglioneuroblastoma: contrasting symptoms of constipation and diarrhoea. Eur $\mathrm{J}$ Pediatr 2003; 162: 511-513.

12. Gera PK, Kikiros CS, Charles A. Chronic diarrhoea: a presentation of immature neuroblastoma. ANZ J Surg 2008; 78: 218-219.

13. Bourdeaut F, de Carli E, Timsit S, Coze C, Chastagner P, Sarnacki S, Delattre O, Peuchmaur M, Rubie H, Michon J. VIP hypersecretion as primary or secondary syndrome in neuroblastoma: A retrospective study by the Société Française des Cancers de l'Enfant (SFCE). Pediatr Blood cancer 2009; 52: $585-590$.

14. Ghishan FK, Soper RT, Nassif ED, Younoszai MK. Chronic diarrhea of infancy: nonbeta islet cell hyperplasia. Pediatrics 1979; 64: 46-49.

15. Balemba OB, Grøndahl ML, Mbassa GK, Semuguruka WD, HaySmith A, Skadhauge E, Dantzer V. The organisation of the enteric nervous system in the submucous and mucous layers of the small intestine of the pig studied by VIP and neurofilament protein immunohistochemistry. J Anat 1998; 192 (Pt 2): 257-267.

16. Said SI, Faloona GR, Harvey S, Deon H. Elevated plasma and tissue levels of vasoactive intestinal polypeptide in the watery diarrhea syndrome due to pancreatic bronchogenic and other tumors. N Engl J Med 1975;293:155-160.

17. Rosenslein BJ, Engelman K. Diarrhoea in a child with a catecholaminesecreting ganglioneuroma case report and review of the literature. J Pediatr 1963;63:217-226.

18. Petersom HD, Collins OD. Chronic diarrhea and failure to thrive secondary to ganglioneuroma. Arch Surg 1967;6:934-936

19. Sackel SG, Manson JE, Harawi SJ, Burakoff R. Watery diarrhea syndrome due to an adrenal pheochromocytoma secreting vasoactive intestinal polypeptide. Dig Dis Sci 1985;30:1201-1207.

20. Smith SL, Slappy AL, Fox TP, Scolapio JS. Pheochromocytoma producing vasoactive intestinal peptide. Mayo Clin Proc 2002;77:97-100. 